\title{
Papulonodular Secondary Syphilis Presenting as Multiple Distinct Cutaneous Lesions in an HIV-Positive Transgender Woman
}

\author{
Kristina Navrazhina Brienne D. Cressey Kira Minkis \\ Department of Dermatology, Weill Cornell Medical College, New York, NY, USA
}

\section{Keywords}

Secondary syphilis $\cdot$ HIV $\cdot$ Papulonodular lesions

\begin{abstract}
We present the first reported case of papulonodular secondary syphilis in an HIV-positive transgender female. Syphilis is classified into primary, secondary, latent, and tertiary stages, with secondary syphilis having notably diverse cutaneous manifestations. Our patient presented with diverse lesions throughout her body, all pathologically consistent with papulonodular secondary syphilis. Proper identification of the multiple presentations of syphilis is crucial to early diagnosis and treatment. This report seeks to broaden the scope of dermatological manifestations that arise secondary to papulonodular syphilis in HIV-positive patients.

(C) 2017 The Author(s) Published by S. Karger AG, Basel
\end{abstract}

\section{Introduction}

Syphilis, defined by primary, secondary, latent, and tertiary stages, is the result of an infection by the microaerophilic spirochete Treponema pallidum, either through sexual contact or congenitally from mother to fetus $[1,2]$. Primary syphilis is characterized by the emergence of a painless red papule that ulcerates into the classic chancre [3]. The chancre commonly appears on the external genitalia of men, or the cervix and labia of women, and is 
usually associated with regional lymphadenopathy $[4,5]$. The primary lesion manifests 2-3 weeks following inoculation. However, many individuals may not notice the painless chancre, which resolves without treatment [4].

Approximately 25\% of untreated individuals develop secondary syphilis, resulting from hematogenous spread of T. pallidum within 4-6 weeks following the appearance of the primary chancre $[3,4,6,7]$. Unlike the indolent lesion of primary syphilis, the generalized mucocutaneous skin lesions associated with secondary syphilis often lead patients to seek clinical attention [2].

\section{Case Report}

A 51-year-old transgender woman with a 20-year history of HIV currently on HAART (most recently CD4 485; viral load 209), diabetes mellitus type 2, hypertension, and asthma presented to the dermatology clinic with hypertrophic, pink, scaly plaques with some crusting, along with several pustules on the trunk, a thin pink-violaceous papule on the right lower leg, and a pink, friable exophytic nodule with a collarette of scale on the right fourth toe (Fig. 1). There was no associated lymphadenopathy. The patient reported that she first noticed the rash 2 weeks prior. At the time of the presentation, she denied fever and chills but reported dry cough and constitutional cold symptoms. Workup at our center included biopsies of the 3 lesions on the left abdomen, right lower leg, and right fourth toe, tissue cultures, wound cultures, and laboratory studies.

Laboratory data were remarkable for an RPR titer of 1:256. All 3 biopsies showed the same fundamental process on histology: psoriasiform and lichenoid dermatitis with significant pustulation, extensive plasmacytosis, and variable granulomatous inflammation. Immunohistochemistry for T. pallidum was positive in all 3 biopsies (Fig. 2). A diagnosis of papulonodular syphilis was established. The patient was treated with 3 weekly doses of long-acting benzathine penicillin G, 2.4 million units intramuscularly. Her lesions subsequently improved and her RPR titer decreased to 1:8 six months following treatment.

\section{Discussion}

Although the cutaneous manifestations are diverse, secondary syphilis typically manifests as symmetrical, nonpruritic, pink, purple, or brown maculopapular eruptions [6, 7]. The characteristic papulosquamous or maculopapular lesions frequently involve the trunk and extremities, with eventual involvement of the palmoplantar surfaces [5]. Patchy alopecia can also occur secondary to involvement of hair follicles in the scalp region $[1,3]$. Previous reports identified follicular, psoriasiform, vesicular, corymbiform, and lichenoid lesions in association with secondary syphilis [3]. Manifestations of secondary syphilis, including cutaneous presentations, are particularly diverse, leading clinicians in the pre-antibiotic era to refer to syphilis as the "great imitator" [2].

Due to its diverse cutaneous manifestations, the differential diagnosis of secondary syphilis includes scabies, drug eruptions, sarcoidosis, lymphoma, pityriasis rosea, leprosy, and atypical mycobacteriosis $[1,3,5]$. The appearance of cutaneous lesions may be paired with systemic symptoms entailing low-grade fever, lymphadenopathy, headache, weight loss, anorexia, sore throat, and malaise [3,4]. The histopathologic analysis of syphilitic lesions is also diverse [6,7]. Typically, the dermis contains superficial and deep perivascular 
inflammatory proliferative changes surrounding the lesions as well as cellular infiltrate consisting of plasma cells, lymphocytes, and histiocytes [3, 7].

Interestingly, while some cutaneous manifestations of syphilis may be similar between HIV-positive and -negative patients, the emergence of atypical and more severe cutaneous presentations of secondary syphilis is more common in HIV-positive individuals [5, 8]. Patients with HIV are more likely to have asymptomatic primary syphilis and are also more likely to initially present clinically with secondary syphilis than HIV-negative individuals [8].

Secondary syphilis presenting as papulonodular lesions is exceedingly rare, with only 15 other cases reported in the past 20 years [7]. As seen in our patient, a previous report noted that the papulonodular form of secondary syphilis spares the palmar and plantar surfaces [9]. However, in contrast to these aforementioned reports, our patient presented with a constellation of simultaneously appearing yet clinically diverse lesions that were all histologically proven to be associated with papulonodular secondary syphilis.

The diagnosis of syphilis is made by a combination of physical examination, histological analysis, and serological tests. Serological diagnosis can be performed as early as 2 weeks from the onset of the initial chancre. In secondary syphilis, both antilipidemic and antitreponemic tests are positive [7]. Benzylpenicillin (penicillin G) remains the current standard treatment for all stages of syphilis [5]. According to the CDC guidelines, HIV-positive individuals with secondary syphilis should be treated the same way as HIV-negative patients, with recommended treatment of a single dose of intramuscular penicillin G (2.4 million units), and response should be confirmed with a 4-fold decline of quantitative nontreponemal titers within 3-6 months of treatment $[1,10]$. However, a multicenter prospective observational a study of 573 HIV-infected individuals showed that a single-dose penicillin G yielded higher serological failure rates compared to 3 weekly doses of penicillin $\mathrm{G}$ that were used to treat our patient [11].

Despite a decline of syphilis towards the end of 20th century, the rates of primary and secondary syphilis have increased by $15.1 \%$ since 2013 [2, 12]. The increase in the disease burden among the population may parallel an increase in atypical syphilitic presentations. The diagnosis of secondary syphilis with atypical manifestations remains challenging. To our knowledge, this is the first reported case of papulonodular syphilis in an HIV-positive transgender woman. Interestingly, this patient presented with multiple distinct lesions which all had the characteristic pathologic findings of papulonodular syphilis, highlighting the diverse presentation of syphilis in this special patient population. Due to the variable cutaneous presentations of secondary syphilis in the context of increasing incidence rates, syphilis should be considered in the differential diagnosis among patients presenting with disseminated papulonodular lesions.

\section{Statement of Ethics}

The authors have no ethical conflicts to disclose.

\section{Disclosure Statement}

The authors have no conflicts of interest to declare. This article has no funding sources and has not been published in any other journal. 


\section{Case Reports in Dermatology}

Navrazhina et al.: Papulonodular Secondary Syphilis Presenting as Multiple Distinct Cutaneous Lesions in an HIV-Positive Transgender Woman

\section{References}

1 Karnath BM: Manifestations of syphilis. Hosp Physician 2009;45:43-48.

-2 Rysgaard C, Alexander E, Swick BL: Nodular secondary syphilis with associated granulomatous inflammation: case report and literature review. J Cutan Pathol 2014;41:370-379.

3 Baughn RE, Musher DM: Secondary syphilitic lesions. Clin Microbiol Rev 2005;18:205-216.

- 4 Little JW: Syphilis: an update. Oral Surg Oral Med Oral Pathol Oral Radiol Endod 2005;100:3-9.

-5 Lautenschlager S: Cutaneous manifestations of syphilis: recognition and management. Am J Clin Dermatol 2006;7:291-304.

-6 Breznik V, Potočnik M, Miljković J: Papulonodular secondary syphilis in a 52-year-old non-HIV heterosexual patient. Acta Dermatovenerol Alp Pannonica Adriat 2010;19:27-30.

7 Veasey JV, Lellis RF, Boin MF, Porto PL, Chen JC: Papulonodular secondary syphilis: a rare clinic presentation confirmed by serologic and histologic exams. An Bras Dermatol 2016;91:205-207. Lynn WA, Lightman S: Syphilis and HIV: a dangerous combination. Lancet Infect Dis 2004;4:456-466.

-9 Baum EW, Bernhardt M, Sams WM, Alexander WJ, Mclean GL: Secondary syphillis. Still the great imitator. JAMA 1983;249:3069-3070.

10 Sexually transmitted diseases: summary of 2015 CDC treatment guidelines. J Miss State Med Assoc 2015;56:34-39.

11 Yang CJ, Lee NY, Chen TC, et al: One dose versus three weekly doses of benzathine penicillin G for patients co-infected with HIV and early syphilis: a multicenter, prospective observational study. PLoS One 2014;9:e109667.

12 Centers for Disease Control and Prevention: Sexually Transmitted Disease Surveillance 2014. Atlanta, Department of Health and Human Services, 2015, pp 29-30.
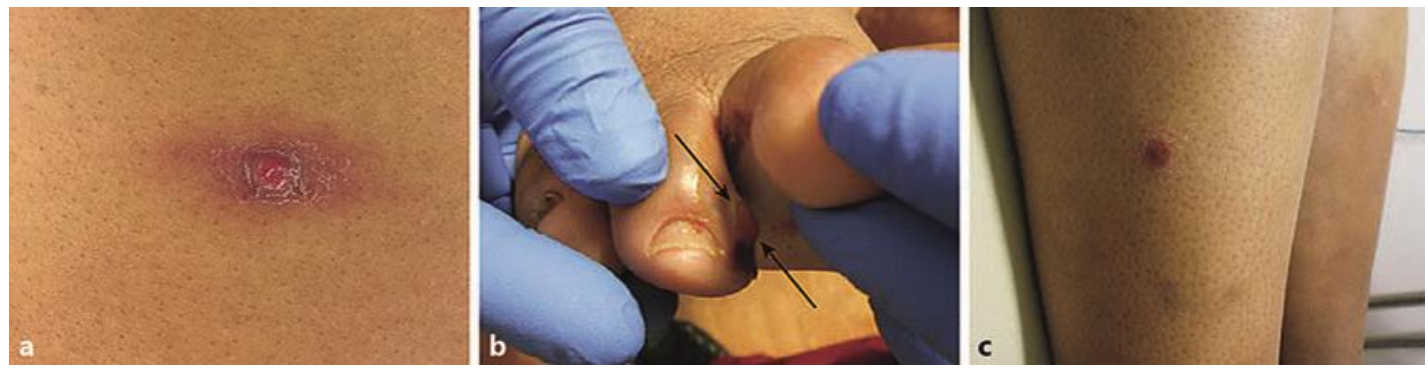

Fig. 1. a Pink, scaly plaque on the left abdomen. b Pink friable nodule on the right fourth toe without any involvement of the plantar toe. c Thin pink-violaceous papule on the right lower leg. 


\section{Case Reports in Dermatology}
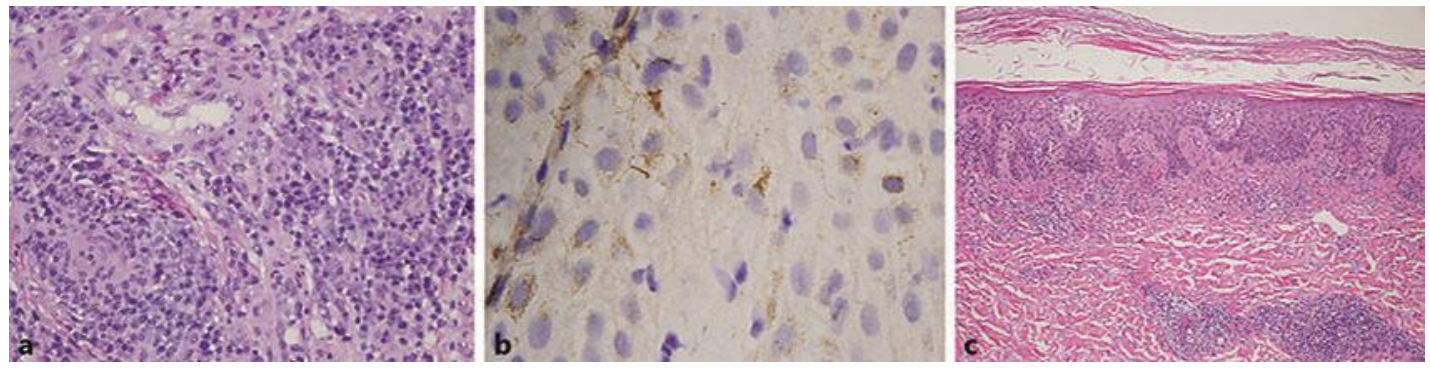

Fig. 2. a Biopsy of the left abdominal lesion showing both superficial and deep dense lymphohistiocytic and plasma cell infiltrate. Hematoxylin-eosin stain. b Immunohistochemistry for T. pallidum demonstrates spirochetes amidst the infiltrate confirming the diagnosis of syphilis. The PASD preparation and Fite stain did not reveal the presence of microbial pathogens. c Biopsy of the lesion on the right lower leg shows psoriasiform epidermal hyperplasia with interface dermatitis. The epidermis is surmounted by a parakeratotic scale. There is perivesicular plasma cell and granulomatous infiltrate. Hematoxylin-eosin stain. Immunohistochemistry for T. pallidum showed the presence of spirochetes within the dermal-epidermal junction. 